\title{
Indirect AMP-Activated Protein Kinase Activators Prevent Incision-Induced Hyperalgesia and Block Hyperalgesic Priming, Whereas Positive Allosteric Modulators Block Only Priming in Mice $^{\mathbb{S}}$
}

\author{
Kufreobong E. Inyang, Michael D. Burton, Thomas Szabo-Pardi, Emma Wentworth, \\ Timothy A. McDougal, Eric D. Ramirez, Grishma Pradhan, Gregory Dussor, \\ and Theodore J. Price
}

School of Behavioral and Brain Sciences, University of Texas at Dallas, Richardson, Texas

Received April 2, 2019; accepted July 16, 2019

\begin{abstract}
AMP-activated protein kinase (AMPK) is a multifunctional kinase that negatively regulates the mechanistic target of rapamycin (mTOR) and mitogen-activated protein kinase (MAPK) signaling, two signaling pathways linked to pain promotion after injury, such as surgical incision. AMPK can be activated directly using positive allosteric modulators, as well as indirectly through the upregulation of upstream kinases, such as liver kinase B1 (LKB1), which is a mechanism of action of metformin. Metformin's antihyperalgesic effects occur only in male mice, raising questions about how metformin regulates pain sensitivity. We used metformin and other structurally distinct AMPK activators narciclasine (NCLS), ZLN-024, and MK8722, to treat incisioninduced mechanical hypersensitivity and hyperalgesic priming in male and female mice. Metformin was the only AMPK activator to have sex-specific effects. We also found that indirect AMPK activators metformin and NCLS were able to reduce mechanical hypersensitivity and block hyperalgesic priming, whereas direct AMPK activators ZLN-024 and MK8722 only blocked priming. Direct and indirect AMPK activators stimulated AMPK in dorsal root ganglion (DRG) neuron cultures to a similar degree; however, incision decreased phosphorylated AMPK (p-AMPK) in DRG.
\end{abstract}

Because AMPK phosphorylation is required for kinase activity, we interpret our findings as evidence that indirect AMPK activators are more effective for treating pain hypersensitivity after incision because they can drive increased p-AMPK through upstream kinases like LKB1. These findings have important implications for the development of AMPK-targeting therapeutics for pain treatment.

\section{SIGNIFICANCE STATEMENT}

Nonopioid treatments for postsurgical pain are needed. Our work focused on whether direct or indirect AMP-activated protein kinase (AMPK) activators would show greater efficacy for inhibiting incisional pain, and we also tested for potential sex differences. We conclude that indirect AMPK activators are likely to be more effective as potential therapeutics for postsurgical pain because they inhibit acute pain caused by incision and prevent the long-term neuronal plasticity that is involved in persistent postsurgical pain. Our work points to the natural product narciclasine, an indirect AMPK activator, as an excellent starting point for development of therapeutics.

\section{Introduction}

Chronic pain is a rapidly growing international health issue that contributes substantially to morbidity, mortality, disability, demands on health care systems, and economic burdens for societies (Institute of Medicine Committee on Advancing Pain Research, Care, and Education, 2011). New classes of analgesics are needed to combat this growing problem

This work was supported by National Institutes of Health grants [R01NS065926] (T.J.P.), [R01NS102161] (T.J.P.), [R01 GM102575] (T.J.P and G.D.), The University of Texas STARS program (T.J.P. and G.D.), and a research funding agreement from CerSci Therapeutics.

https//doi.org/10.1124/jpet.119.258400.

S This article has supplemental material available at jpet.aspetjournals.org.
(Finnerup et al., 2015). One of the most common and significant forms of chronic pain is chronic postsurgical pain (Haroutiunian et al., 2013), which causes major functional impairment in 5\%-10\% of surgery patients (Kehlet et al., 2006). Postsurgical pain medicines that can provide pain relief acutely and diminish the probability of developing chronic pain would improve health care (Price et al., 2018). AMP-activated protein kinase (AMPK) is a cellular fuelsensing enzyme present in most cells across species (Hardie, 2007). This kinase detects changes in the AMP/ATP ratio to regulate anabolic processes when cellular energy status is low (Kahn et al., 2005). Several studies have shown that AMPK plays a key role in nociceptive sensitization (Song et al., 2015; Bullón et al., 2016). AMPK activation causes

ABBREVIATIONS: ACC, acetyl-CoA carboxylase; AMPK, AMP-activated protein kinase; DRG, dorsal root ganglion; ICR, Institute of Cancer Research; LKB1, liver kinase B1; MAPK, mitogen-activated protein kinase; mTOR, mechanistic target of rapamycin; NCLS, narciclasine; PGE 2 , prostaglandin $\mathrm{E}_{2} ; \mathrm{p}$, phosphorylated; $\mathrm{SNI}$, spared nerve injury. 
a decrease in mechanistic target of rapamycin (mTOR) and MAPK signaling, leading to many cellular effects, including suppression of protein synthesis mediated by activation of mTOR and MAPK pathways (Hardie, 2007). In animal models of incisional and nerve injury pain, MAPK and mTOR signaling pathways are turned on in dorsal root ganglion (DRG) neurons, including nociceptors, and these signaling events are a causative factor in increased nociceptor excitability and behavioral nociceptive hypersensitivity (Sato and Ohshima, 2000; Banik et al., 2005). Many groups have shown that AMPK activators can be effective in attenuating this cellular hyperexcitability and behavioral hypersensitivity (Tillu et al., 2012; Mejia et al., 2016). We have also shown that AMPK activators prevent the development of hyperalgesic priming, a preclinical model that mimics aspects of the transition from acute to chronic pain.

Metformin is a commonly prescribed drug for type 2 diabetes that activates AMPK indirectly in a liver kinase B1 (LKB1)-dependent fashion (Shaw et al., 2005). Although the exact mechanism of the drug remains an area of active inquiry, metformin is safe and potentially could be repurposed as a pain-relieving drug (Rena et al., 2017; Wu et al., 2018). We have previously shown that metformin is effective in reversing spared nerve injury (SNI)-induced neuropathic pain (Melemedjian et al., 2011), as well as reducing mechanical hypersensitivity after surgical incision without reducing the rate of wound healing (Burton et al., 2017). Interestingly, the antihyperalgesic effects of metformin seem to occur specifically in male mice (Inyang et al., 2019).

Various other AMPK activators have emerged in recent years and may be better potential analgesics than metformin (Cao et al., 2018; Qin et al., 2018). It is also unknown whether these drugs could show sex-specific effects. Narciclasine (NCLS) is a natural compound from the plant Amaryllidaceae (Dumont et al., 2007) that indirectly activates AMPK (Julien et al., 2017) through a mechanism that may involve cAMP (Zhang et al., 2009). NCLS and related compounds are also known to have anti-inflammatory effects (Yui et al., 2001; Lubahn et al., 2012). Whereas indirect AMPK activators stimulate upstream kinases that phosphorylate AMPK, another way to activate AMPK is via direct positive allosteric mechanisms. Several positive allosteric modulators of AMPK, including ZLN024 (Zhang et al., 2013) and MK8722 (Feng et al., 2017; Weihrauch and Handschin, 2018), have recently been described and have favorable pharmacokinetics for in vivo dosing.

The goal of the experiments described here was to establish whether the sex specificity seen with metformin treatment after SNI was also found in the incisional pain model. We also sought to determine whether other AMPK activators can be effective in alleviating incision-induced nociceptive hypersensitivity and hyperalgesic priming in male and/or female mice. Our experiments demonstrate that metformin is effective in reducing hypersensitivity after an incision, as well as hyperalgesic priming in male but not in female mice. On the other hand, NCLS reduced acute pain caused by incision and blocked development of hyperalgesic priming in male and female mice. Direct AMPK allosteric modulators ZLN024 and MK8722 both attenuated hyperalgesic priming but were effective in both sexes. We conclude that indirect AMPK activators have the best potential for future development as pain therapeutics.

\section{Materials and Methods}

Laboratory Animals. Animal procedures were approved by The University of Texas at Dallas Institutional Animal Care and Use Committee and were in accordance with National Institutes of Health Guidelines. All the experiments were performed on male or female Institute of Cancer Research (ICR) outbred mice obtained from Envigo (Cambridgeshire, UK) at 4 weeks of age or bred at the University of Texas at Dallas. Mice were housed in the University of Texas at Dallas Animal Care Facility for at least 1 week before the start of behavior testing and surgery. Animals had ad libitum access to food and water and were on a 12-hour noninverted light/dark cycle. Experimenters were blinded to treatment groups in behavioral experiments. Mice were randomized to treatment groups using a random number generator and in such a manner that multiple treatment groups were always found within any individual cage of animals. Male and female mice were housed separately in groups of four per cage.

Behavioral Testing. The plantar incision model was used to induce postsurgical pain in mice as described previously (Banik et al., 2006). Mechanical sensitivity was assessed using stimulation of the hindpaw of the mouse with calibrated von Frey filaments from Stoelting Co. (Wood Dale, IL). We used 0.6-, 1.0-, and 1.4-g filaments and measured the response frequency to 10 consecutive stimulations of the hindpaw with each filament; stimulations were spaced by at least 5 seconds after 45 minutes of habituation to the testing boxes. The number of responses for each filament force was recorded. After baseline testing, mice were treated systemically with an AMPK activator for seven consecutive days. Immediately after day 3 of treatment, plantar incision surgery was performed by making a 5 -mm longitudinal incision with a number 11 scalpel blade in the skin of the left hindpaw and the underlying muscle tissue $2 \mathrm{~mm}$ below the heel. The wound was closed using a 5-mm silk suture, followed by a $200-\mu \mathrm{l}$ subcutaneous injection of gentamicin $(5 \mathrm{mg} / \mathrm{ml}$; Sigma-Aldrich, St. Louis, MO). For the sham surgery, mice were put under isoflurane for 5 minutes, followed by subcutaneous gentamicin. Mice were tested for mechanical hypersensitivity periodically until response frequency returned to baseline levels. After the return to baseline, hyperalgesic priming was tested by giving each animal an intraplantar injection of prostaglandin $\mathrm{E}_{2}\left(\mathrm{PGE}_{2}\right)(100 \mathrm{ng} / 25 \mu \mathrm{l})$. Response frequency after $\mathrm{PGE}_{2}$ was tested 3 and 24 hours postinjection. The different AMPK activators used in this experiment were metformin i.p. ( $200 \mathrm{mg} / \mathrm{kg}$; LKT Laboratories,St. Paul, MN), ZLN-024 hydrochloride i.p. (30 mg/kg; Tocris, Bristol, UK) (Zhang et al., 2013), NCLS p.o. (3 mg/kg; Santa Cruz Biotechnology, Inc., Dallas, TX) (Julien et al., 2017), and MK-8722 p.o. (a gift from Merck \& Co., Kenilworth, NJ), 30 mg/kg) (Feng et al., 2017). Metformin and ZLN were dissolved in $0.9 \%$ saline, and NCLS was made in (2-hydroxypropyl)- $\beta$-cyclodextrin (Sigma Aldrich). MK-8722 was administered in $0.25 \%$ methyl cellulose, $5 \%$ Tween-80, and $0.02 \%$ sodium dodecyl sulfate (Feng et al., 2017).

Rotorod testing was done using a series 8 rotorod device from IITC Life Science, Inc. (Woodland Hills, CA). Mice were placed on the rotorod with the setting of four rotations per minute to start with an increase to 40 rotations per minute over the course of 108 seconds. The latency to fall was measured using the device. Testing was done before drug treatment and again on the last day of drug treatment.

Metformin Pharmacokinetics. This study was done at Sai Life Science Limited at Hinjewadi, India. Eighteen ICR mice weighing between 20 and $25 \mathrm{~g}$ were used, nine males and nine females. Blood samples of approximately $60 \mu \mathrm{l}$ were collected under light isoflurane anesthesia from sets of three male and female mice at $0.5,1$, and 4 hours after dosing. Plasma was harvested by centrifugation of blood and stored at $-70^{\circ} \mathrm{C}$ until analysis. After blood collection, brain samples were isolated at each time point from the same animals. Brains was dipped three times in ice-cold phosphate-buffered saline, blotted dry, and weighed. Brain samples were homogenized using icecold phosphate-buffered saline with twice the volume of brain weight and then stored at $-70^{\circ} \mathrm{C}$ until analysis. Plasma and brain samples were quantified by fit-for-purpose liquid chromatography-tandem 
mass spectrometry methods. Data are shown as nanograms per milliliter in plasma and nanograms per gram of brain weight.

Neuron Culture. DRGs were extracted aseptically from 4-weekold male ICR mice in Hanks' buffered salt solution (HBSS; Invitrogen, Waltham, MA) on ice. The DRGs were dissociated enzymatically at $37^{\circ}$ C, first with collagenase A $(1 \mathrm{mg} / \mathrm{ml}$, Roche Diagnostics, Basel, Switzerland) for 25 minutes and then collagenase D ( $1 \mathrm{mg} / \mathrm{ml}$; Roche) that included papain ( $30 \mu \mathrm{g} / \mathrm{ml}$; Roche) for 20 minutes. Afterward, a trypsin inhibitor $(1 \mathrm{mg} / \mathrm{ml}$; Roche) that contained bovine serum albumin (bovine serum albumin, $1 \mathrm{mg} / \mathrm{ml}$; Fisher) was applied, and the ganglia were mixed to allow further dissociation with a polished Pasteur pipette. The tissue was then filtered through $70-\mu \mathrm{m}$ nylon cell strainer (Falcon; Corning, NY) and resuspended in Dulbecco's modified Eagle's medium F-12 GlutaMax media (Invitrogen) that contained $10 \%$ fetal bovine serum (fetal bovine serum; Hyclone Laboratories, Inc., South Logan, UT) and $1 \times$ penicillin streptomycin
(Pen-Strep; Invitrogen). The media also contained serve growth factor (10 ng/ml; Millipore, Billierca, MA) and 5-fluoro-2'-deoxyuridine + uridine $(3.0 \mu \mathrm{g} / \mathrm{ml}+7.0 \mu \mathrm{g} / \mathrm{ml}$; Sigma) to reduce proliferation of glia and fibroblasts. Neurons were cultured for 7 days on 12-mm glass coverslips (no. 1 thickness; Chemglass Life Sciences, Inc., Vineland, NJ) in a 24-well tissue culture plate (Falcon) coated with poly-D-lysine (Sigma) at $37^{\circ} \mathrm{C}$ with $95 \%$ air and $5 \% \mathrm{CO}_{2}$. On the day of the experiment, drugs were diluted into Dulbecco's modified Eagle's medium F-12 plus GlutaMax media and added directly onto the neurons at concentrations indicated in the results for 1 hour. All DRGs from one mouse were used to generate approximately four coverslips of primary cells. Coverslips from multiple independent animals were used in each experiment.

Immunocytochemistry and Digital Image Analysis. After AMPK activator treatment, the cells were washed with phosphatebuffered saline and fixed with $10 \%$ formalin in phosphate-buffered saline for 30 minutes. Cells were blocked with $10 \%$ normal goat serum
A
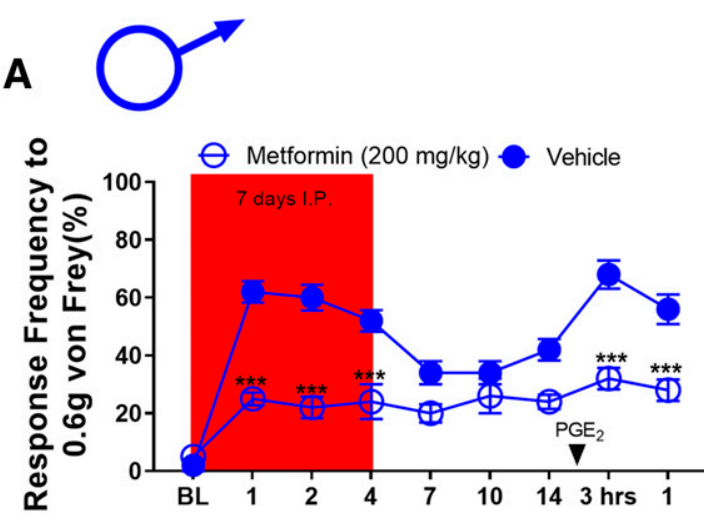

B

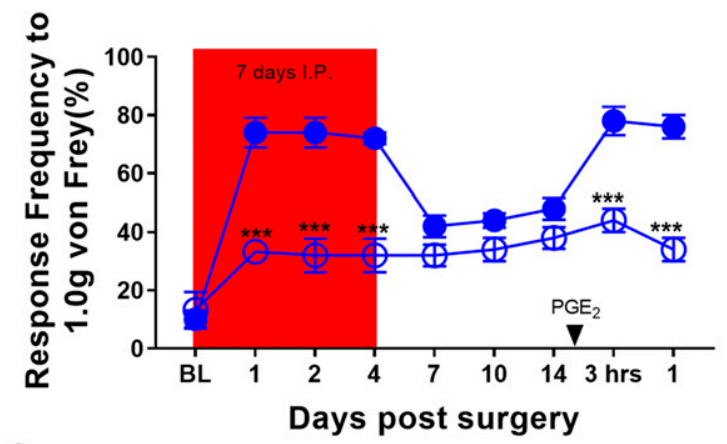

C

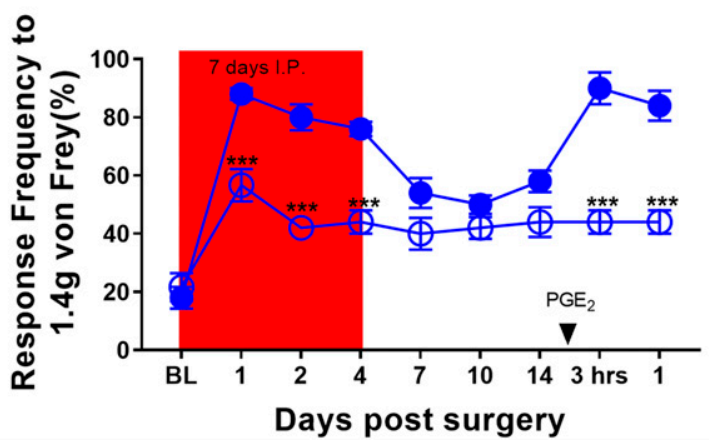

D<smiles>[C]1CCCCC1</smiles>
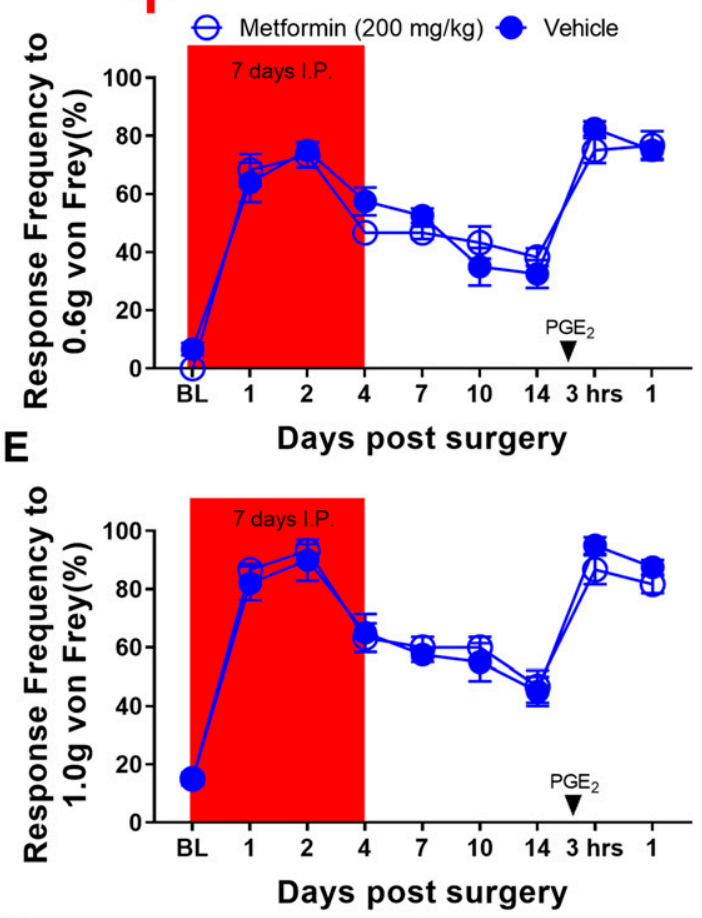

$\mathbf{F}$

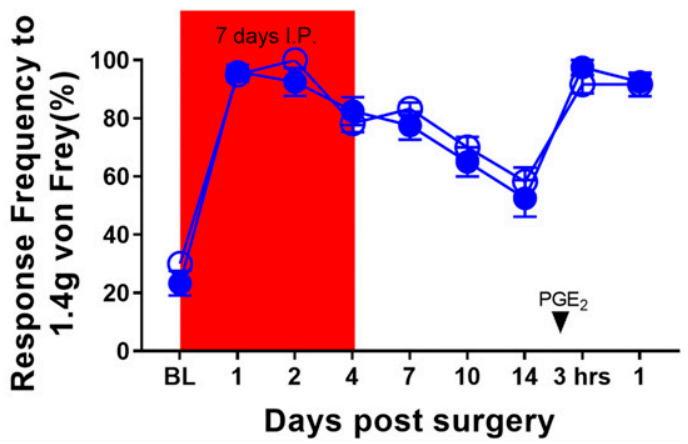

Fig. 1. Metformin treatment attenuates incision-induced mechanical hypersensitivity and blocks hyperalgesic priming in male mice: (A-C). Metformin treatment starting immediately after baseline measurements, which were done 3 days before plantar incision, decreased mechanical hypersensitivity

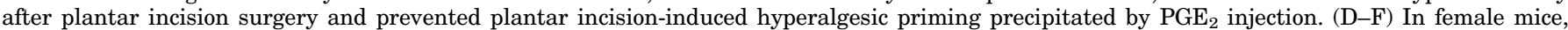
metformin treatment did not decrease mechanical hypersensitivity compared with vehicle-treated mice. Metformin also failed to prevent plantar incision-induced hyperalgesic priming precipitated by $\mathrm{PGE}_{2}$ injection. $* * * P<0.001$; treatment effect; $n=6$ for the metformin groups and $n=5$ for the vehicle groups. 


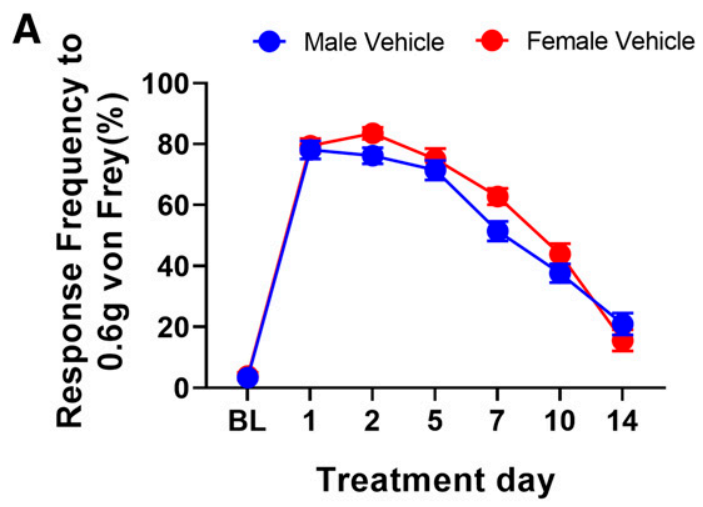

B
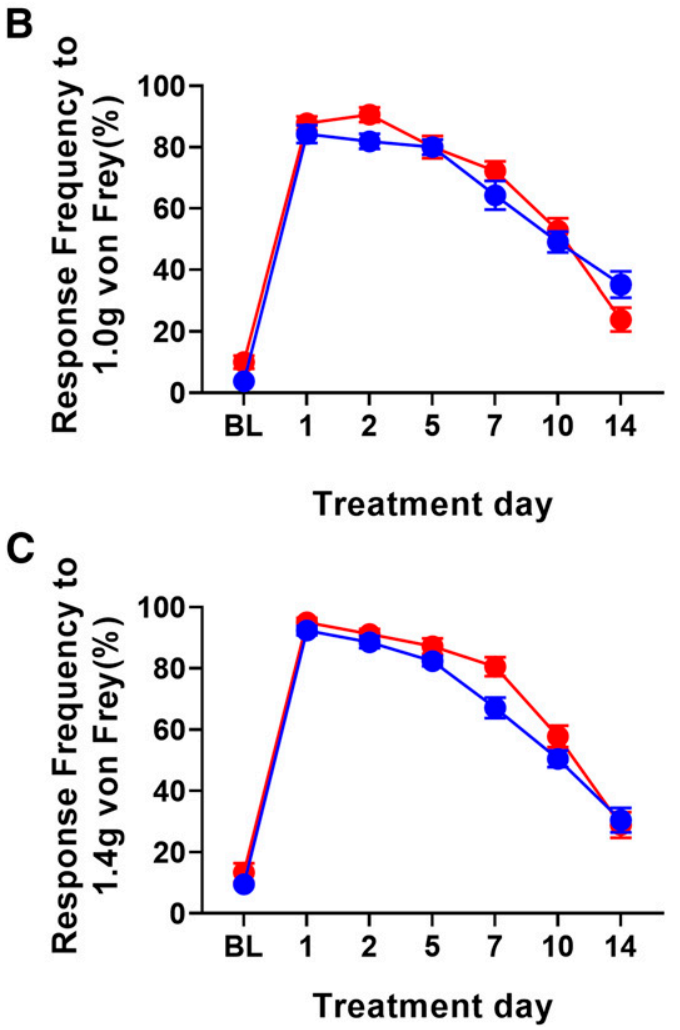

Fig. 2. Male and female mice recover from plantar incision surgery at the same rate. (A-C) Plantar incision surgery in male and female mice caused an increase in mechanical hypersensitivity that resolved at the same rate with no significant differences in mechanical hypersensitivity. $n=21$ males and $n=18$ females.

and labeled with anti-peripherin, mouse monoclonal (1:500; Sigma) and phospho-acetyl-CoA carboxylase (ACC) (Ser 79) (p-ACC, 1:1000; catalog no. 3661; Cell Signaling Technologies) overnight at $4^{\circ} \mathrm{C}$. Next, cells were washed and incubated with flurochrome-conjugated secondary antibodies (Alexa Fluor, antirabbit 488 and anti-mouse 568; Invitrogen) and counterstained with a DNA stain, 4' ${ }^{\prime}$,6-diamidino-2-phenylindole (DAPI) (Invitrogen) and mounted with Prolong Gold (Invitrogen). Images were taken on an Olympus Fluoview FV1200 laser scanning confocal microscope and analyzed using the colocalization tool within Olympus FV software. The intensity of each channel was adjusted so that only areas that contained a strong signal of 488 and $405 \mathrm{~nm}$ were visible. This adjusted imaged contained distinct puncta that could then be counted and analyzed using GraphPad Prism (GraphPad Software, San Diego, CA). Results were reported as the average percent area of p-ACC signal in neurons labeled with peripherin for all representative pictures.
Western Blotting. Male mice were anesthetized with isoflurane and spinal cords and DRGs innervating the hindpaw (L3-L5) were removed. Fresh tissues were placed in ice cold lysis buffer $(50 \mathrm{mM}$, Tris pH 7.4, $150 \mathrm{mM} \mathrm{NaCl}, 1 \mathrm{mM}$ EDTA pH 8.0, and $1 \%$ Triton X100) containing protease and phosphatase inhibitors cocktails (Sigma-Aldrich) and homogenized using beaded homogenization tubes (Bertin Corp; Rockville, MD.). Samples were centrifuged at $14,000 \mathrm{rpm}$ for 15 minutes at $4^{\circ} \mathrm{C}$, and the supernatant containing protein extracts was collected. Protein concentrations were assessed using the Pierce BCA protein assay kit (ThermoFisher Scientific) as directed. A total of $10 \mu \mathrm{g}$ of protein was mixed with Laemmli sample buffer (Bio-Rad, Hercules, CA) and 2-mercaptoethanol and was heated at $95^{\circ} \mathrm{C}$ for 5 minutes. Samples were loaded into each well of a $10 \%$ SDS-PAGE gel along with $15 \mu \mathrm{l}$ of Precision plus protein kaleidoscope prestained protein standards (Bio-Rad). Proteins were transferred to a 0.45 PVDF membrane (Millipore) at $100 \mathrm{~V}$ for 1 hour. Membranes were blocked using 5\% nonfat dry milk in $1 \times$ Trisbuffered saline-Tween for 2 hours before primary antibody incubation. Primary antibodies used for this experiment were pAMPK $\alpha$ (Thr 172) (1:1000, catalog no. 2535; Cell Signaling Technology, Danvers, MA) and total AMPK $\alpha$ (1:1000, catalog no. 2532; Cell Signaling). Bands were visualized using a Bio-Rad ChemiDoc Touch. Analysis was performed using Image Laboratory version 6.0; BioRad, Hercules, CA.

Statistics. Data are shown as mean \pm S.E.M., and the numbers of animals or samples used in each analysis are given in the figure legends. GraphPad Prism 7 was used to analyze data and to do curve fitting, and other statistical tests are given in the figure legends. Two-way analysis of variance (ANOVA)s were used to analyze von Frey data. Post-hoc tests used were the Bonferroni's multiple comparisons test. Significance level was set at $\alpha<0.05$. Details on test statistics are given in Supplemental Table 1.

\section{Results}

Metformin Decreases Incision-Induced Mechanical Hypersensitivity and Blocked Hyperalgesic Priming in Male but Not in Female Mice. To assess the effects of metformin on incision-evoked mechanical hypersensitivity in male mice, we first obtained baseline responses for von Frey filament strengths of $0.6,1.0$, and $1.4 \mathrm{~g}$. After baseline, mice were allocated into metformin $(200 \mathrm{mg} / \mathrm{kg})$ and vehicle (0.9\% saline) interperitoneal treatment groups for 7 days. On day 3 of treatment, plantar incision surgery was performed on all the mice. These mice were tested periodically over the next 2 weeks until they returned to baseline. Once the mice returned to baseline-level sensitivity, animals received $100 \mathrm{ng} \mathrm{PGE}_{2}$ into the affected hindpaw to assess hyperalgesic priming. These mice were tested 3 and 24 hours postinjection. In male mice, metformin reduced acute mechanical hypersensitivity after plantar incision. Metformin also prevented the development of hyperalgesic priming (Fig. 1, A-C). These findings are consistent with our previously published data using the up-down von Frey testing method (Burton et al., 2017). A separate group of sham surgery male mice were allocated into metformin and vehicle treatment groups and baselined for von Frey and rotarod testing to assess possible effects on motor behavior. These mice were treated for 7 days and given a sham surgery on day 3 . The mice were tested periodically for von Frey responses, and no differences were observed based on treatment (Supplemental Fig. 1, A-C). After the last day of drug treatment, these mice were tested on the rotarod again to assess the effects of metformin on motor behavior. Metformin had no effect on locomotive behavior in male mice (Supplemental Fig. 1D). 
To test whether similar effects would be observed in female mice, we obtained baseline mechanical sensitivity measurements and randomized them into metformin and vehicle treatment groups under conditions similar to those of males. On day 3 of treatment, plantar incision surgery was performed on these mice. Mechanical hypersensitivity was tested $1,2,4,7,10$, and 14 days postsurgery. Once the mice returned to baseline level sensitivity, animals received $100 \mathrm{ng}$ of $\mathrm{PGE}_{2}$ into the affected hindpaw. Unlike with male mice, metformin had no effect on the acute hypersensitivity caused by incision. Metformin also failed to block hyperalgesic priming in female mice (Fig. 1, D-F). These findings are consistent with our observations in female SNI mice; metformin relieved mechanical hypersensitivity in only male mice. Although a pronounced difference in mechanical hypersensitivity was seen between male and female metformin-treated mice after plantar incision, we did not observe any sex difference in the duration or magnitude of mechanical hypersensitivity in vehicle-treated mice (Fig. 2). To test the possibility that lower doses of metformin could be effective in female mice in the plantar incision test, we did treatments with 100 and $50 \mathrm{mg} / \mathrm{kg}$ metformin. These doses also failed to impact acute mechanical hypersensitivity or to block hyperalgesic priming in female mice (Supplemental Fig. 2).

We next sought to determine whether this sex difference with metformin could be explained by pharmacokinetics. Nine male and nine female ICR mice were given a single i.p. dose of $200 \mathrm{mg} / \mathrm{kg}$ metformin. A third of the mice were euthanized 30 minutes after injection, another third 1 hour
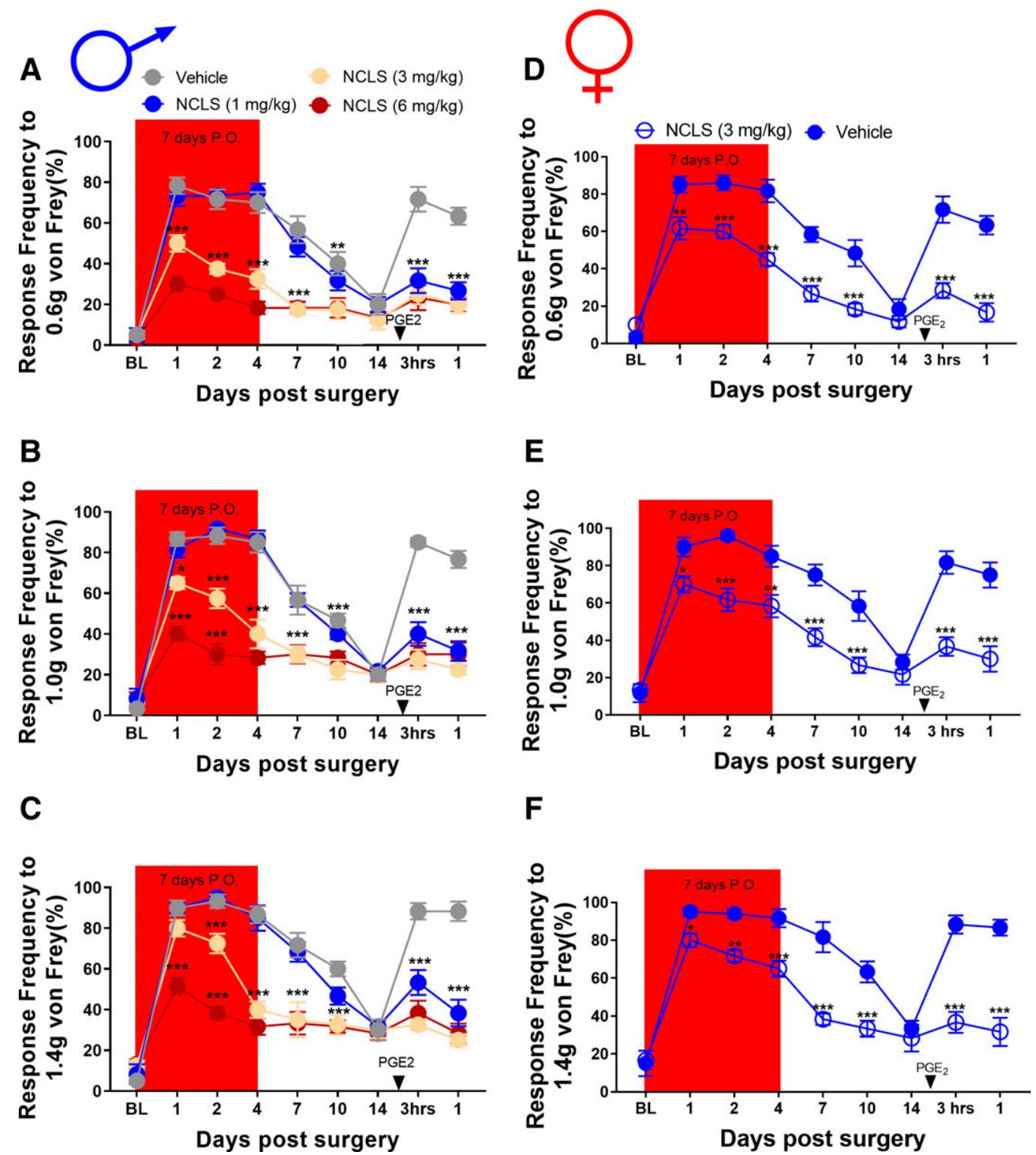

Fig. 3. NCLS treatment attenuates surgery-induced mechanical hypersensitivity and blocks hyperalgesic priming in male and female mice: (A-C). NCLS treatment starting immediately after baseline measurements dose dependently decreased mechanical hypersensitivity after plantar incision surgery and prevented plantar incision-induced hyperalgesic priming precipitated by $\mathrm{PGE}_{2}$ injection at all doses. ${ }^{*} P<0.05 ; * * P<0.01 ; * * * P<0.001$; $n=4$ for the NCLS groups, $n=3$ for the vehicle group. (D-F) NCLS treatment also decreased mechanical hypersensitivity after plantar incision surgery and prevented surgery-induced hyperalgesic priming precipitated by $\mathrm{PGE}_{2}$ injection. ${ }^{*} P<0.05 ; * * P<0.01 ; * * * P<0.001 ; n=6$ for the NCLS group, $n=$ 6 for the vehicle group. 
after injection, and the remaining 4 hours after injection; brain and plasma were taken at each time point. Metformin was measured in the brain and plasma of each animal and compared for each timepoint and across sexes. Surprisingly, we found that plasma levels of metformin were higher in female mice at 0.5 and 1 hour after intraperitoneal injection (Supplemental Fig. 3A). Brain levels were higher in female mice at all time points (Supplemental Fig. 3B). Brain-toblood ratios did not differ between sexes (Supplemental Fig. 3C; Supplemental Table 2). Differences in pharmacokinetics for plasma or blood levels of metformin do not explain the sex differences in efficacy seen in the incisional model.
NCLS Decreased Incision-Induced Mechanical Hypersensitivity and Blocked Priming in Male and Female Mice. We then tested whether a structurally distinct AMPK activator would show similar sex-specific effects. We obtained baseline mechanical sensitivity measurements from male mice and then sorted them into $\operatorname{NCLS}(1,3$, or $6 \mathrm{mg} / \mathrm{kg})$ and vehicle ((2-hydroxypropyl)- $\beta$-cyclodextrin) groups for oral administration. These mice were treated orally for 7 consecutive days, and plantar incision surgery was performed on day 3 of treatment. These mice were tested for von Frey responses under the same time course as in the metformin experiments. Once the mice returned to baseline sensitivity,
A

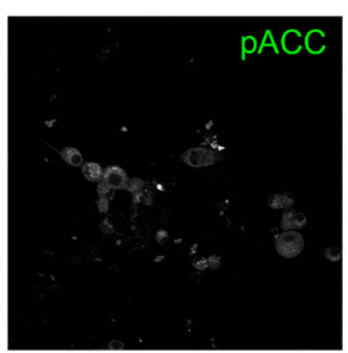

B

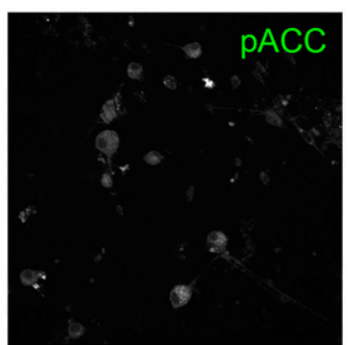

C

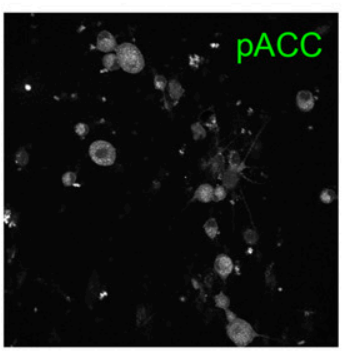

Vehicle

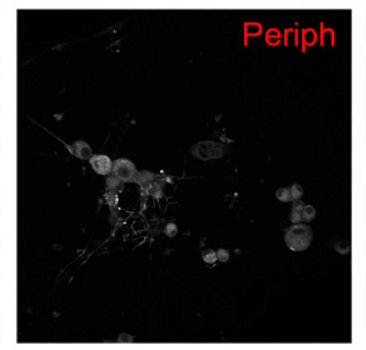

$100 \mathrm{nM}$ NCLS
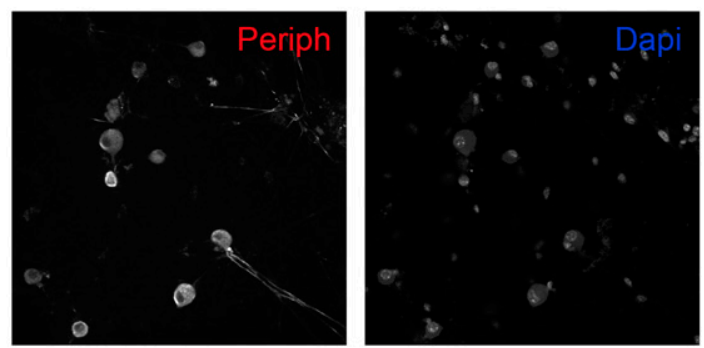

1 uM NCLS
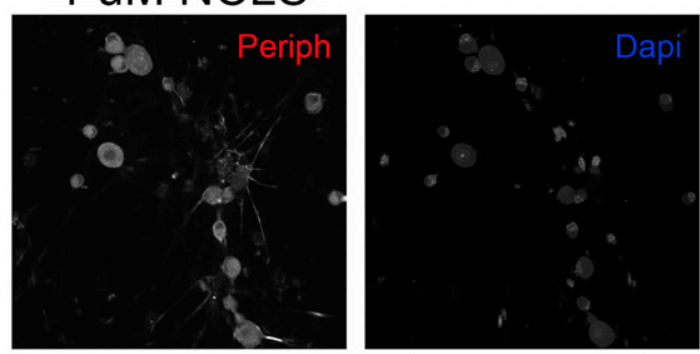

Fig. 4. Narciclasine induces AMPK activity in male DRG neurons in vitro. Male neuron cultures were treated with vehicle (A), $100 \mathrm{nM}$ (B), or $1 \mu \mathrm{M}$ NCLS (C) for 1 hour. Representative immunohistochemistry images of the DRG neurons at $40 \times$ magnification. Quantification of images shown in (D). One micromolar NCLS increased p-ACC intensity in neuron cultures. Only neurons that were positive for peripherin staining were analyzed. Maximum florescence refers to the maximum florescence intensity per neuron analyzed. $* * P<0.01 . n=39$ images analyzed per group.
D

\section{$1 \mathrm{hr}$ NCLS}

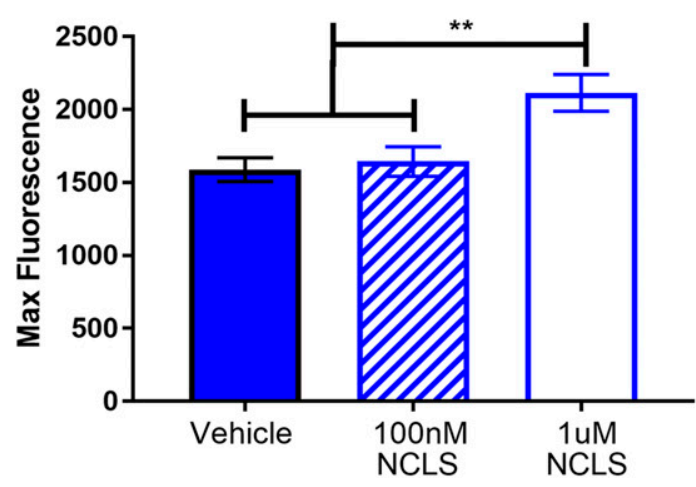


animals received $\mathrm{PGE}_{2}$ into the hindpaw and were tested 3 and 24 hours after injection. In male mice, NCLS reduced the mechanical hypersensitivity after plantar incision in a dosedependent fashion. All NCLS doses completely blocked development of hyperalgesic priming (Fig. 2, A-C). Because the $3-\mathrm{mg} / \mathrm{kg}$ dose showed full efficacy in male mice, we chose to continue our experiments in female mice using only this dose. The experiment in male mice was repeated in its entirety in a group of female mice over the same time course. Once the females returned to baseline, they also received $\mathrm{PGE}_{2}$ into the affected paw and were tested 3 and 24 hours after surgery. Like the experiment with males, NCLS prevented acute mechanical hypersensitivity in female mice and blocked development of hyperalgesic priming (Fig. 2, D-F). Therefore, indirect AMPK activators can be effective in reducing incision-evoked mechanical hypersensitivity and hyperalgesic priming in both male and female mice. A separate group of sham surgery male mice were allocated into NCLS and vehicle treatment groups and baselined for von Frey and rotarod testing to assess possible effects on motor behavior. These mice were also treated for 7 days but given a sham surgery treatment on day 3 . The mice were tested periodically for von Frey responses, and no differences were observed based on treatment (Supplemental Fig. 4, A-C). NCLS also had no effect on rotorod behaviors (Supplemental Fig. 4D).

Whereas NCLS has been shown to activate AMPK in other cell types, it has never been tested for AMPK activity in DRG neurons. To assess this directly, DRGs were cultured from naïve male mice and treated with vehicle, $100 \mathrm{nM}, 1 \mu \mathrm{M}$ NCLS
A

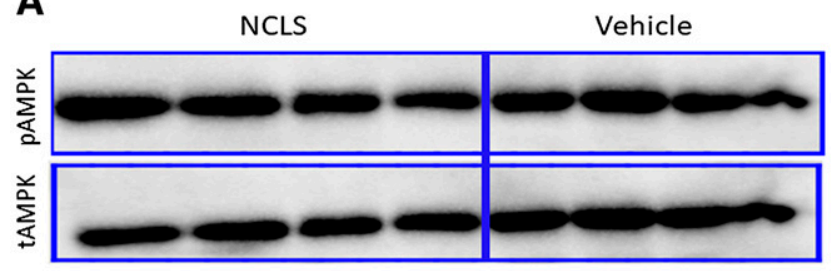

B

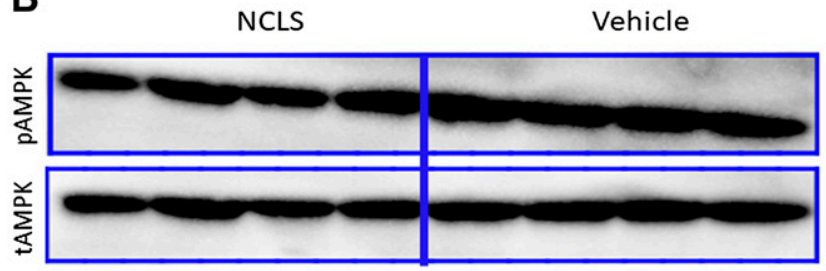

C

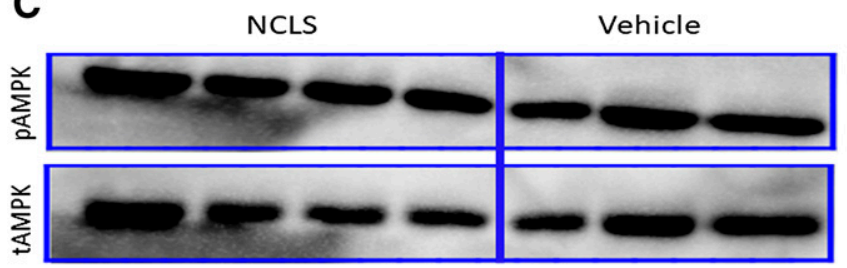

D

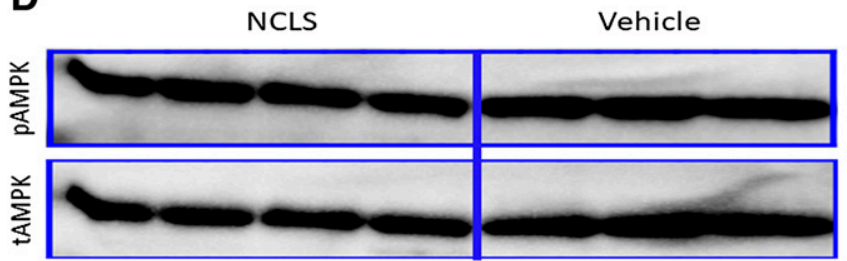

E

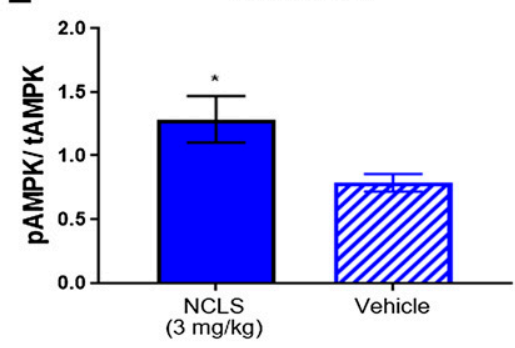

$\mathbf{F}$

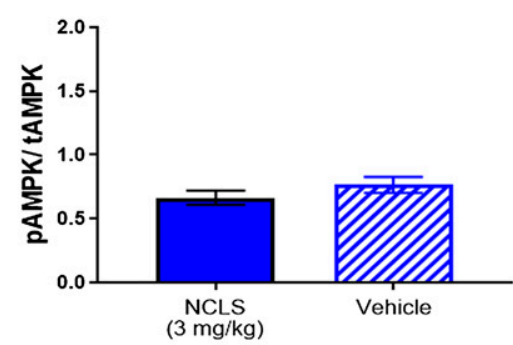

G Female NCLS DRG

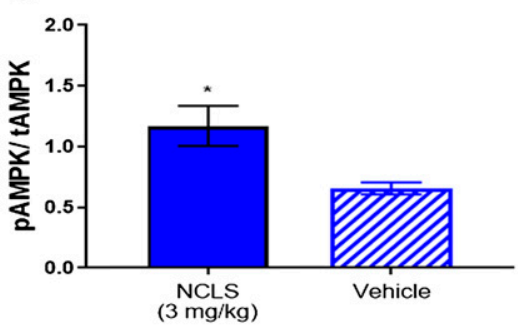

H Female NCLS Liver

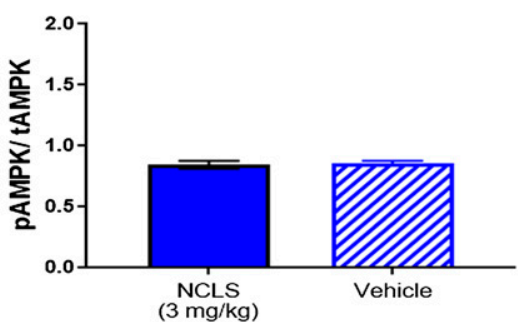

Fig. 5. Narciclasine increases AMPK signaling in male and female DRGs. A single dose of NCLS (3 mg/kg) caused a significant increase in AMPK signaling in DRGs for male (A) and female (C) mice 1 hour after injection but did not affect AMPK signaling in liver for males (B) or females (D). NCLS treatment increased the ratio of p-AMPK to total AMPK in male (E) and female (G) DRGs but not in male (F) or female $(\mathrm{H})$ livers. *P $<0.05$; Student's $t$ test; $n=4$ per condition in male mice; $n=4$ for female NCLS treatment, $N=3$ for female vehicle. 
for 1 hour. We observed a significant increase in p-ACC intensity with NCLS treatment at $1 \mu \mathrm{M}$, demonstrating that NCLS induces AMPK activation in DRG neurons (Fig. 3, A-D). This concentration is higher than previous demonstrations of AMPK activation with $20 \mathrm{nM}$ NCLS in skeletal muscle cells, but those experiments were done over 48 hours of treatment and ours was done with a 1-hour treatment. The discrepancy in the concentration of NCLS needed to activate AMPK may be cell type- or time course-dependent. We conclude from this biochemical study that NCLS is a potent activator of AMPK in DRG neurons.

In addition to these in vitro findings, we used Western blotting to confirm that NCLS can activate AMPK in the DRG in males and females in vivo. Mice of both sexes were given a single dose of NCLS (3 mg/kg) and DRGs, and livers were taken 1 hour postinjection and homogenized for Western blotting. Primary antibodies used were p-AMPK and total AMPK to assess the effects of NCLS on AMPK signaling in these tissues. We observed a significant increase in p-AMPK/ total AMPK ratio in both the male and female DRGs with
NCLS treatment compared with vehicle (Fig. 4). No difference was found in the $\mathrm{p}$-AMPK/total AMPK ratio in male or female livers with NCLS treatment, which is consistent with previously published data with this compound (Julien et al., 2017).

Direct Allosteric AMPK Activators Reduce Hyperalgesic Priming in Male and Female Mice without Impacting Acute Incision-Induced Mechanical Hypersensitivity. Metformin and NCLS activate AMPK via upstream signaling mechanisms. ZLN024 and MK8722 are structurally distinct AMPK activators that bind directly to the kinase to allosterically increase kinase activity, albeit via distinct mechanisms. We used these compounds because they have thoroughly described pharmacokinetics. After establishing baseline mechanical sensitivity measurements from male mice, we then assigned them to ZLN-024 (30 mg/kg) and vehicle $(0.9 \%$ saline $)$ i.p. treatment groups. These mice received intraperitoneal ZLN-024 for 7 consecutive days, and plantar incision surgery was performed on day 3 of treatment. Once the mice returned to baseline sensitivity, animals
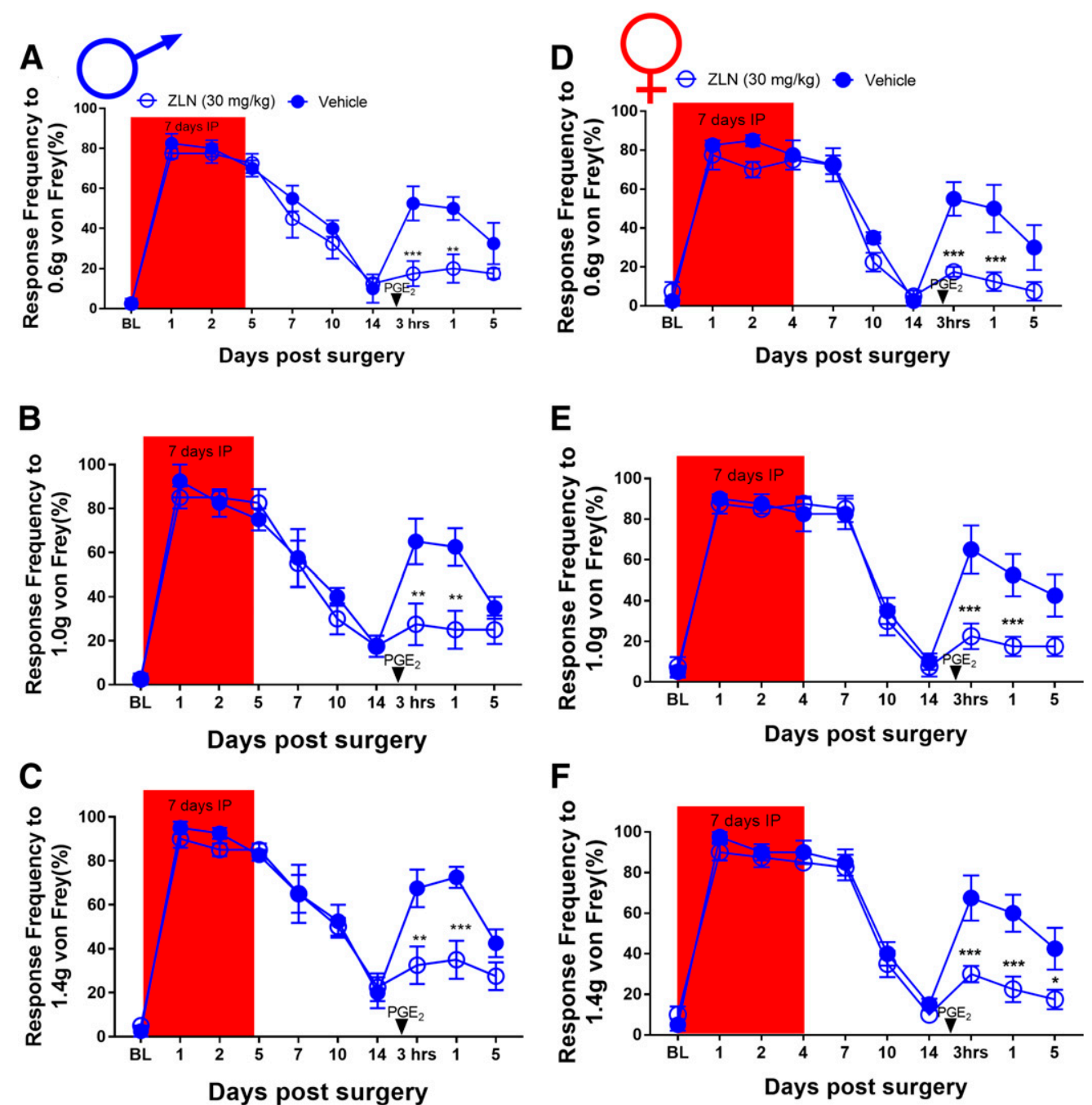

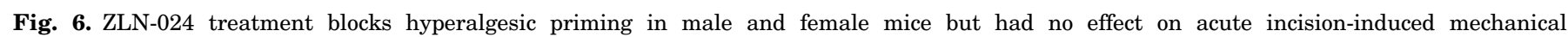

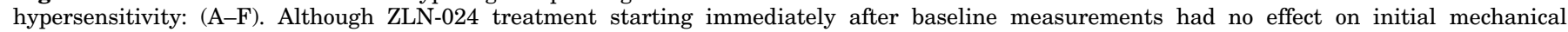

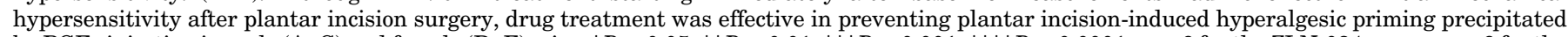

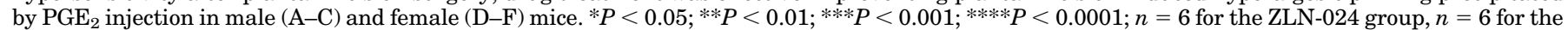
vehicle group. 
received $\mathrm{PGE}_{2}$ into the hindpaw and were tested 3 and 24 hours after injection. In male mice, ZLN-024 did not significantly decrease mechanical hypersensitivity after plantar incision surgery, but 7-day treatment did block hyperalgesic priming (Fig. 5, A-C). For the female cohorts, ZLN-024 also had no effect on the initial surgery-induced mechanical hypersensitivity, but it did block hyperalgesic priming after $\mathrm{PGE}_{2}$ just as with the male cohort (Fig. 5, D-F). Using the same paradigm for testing in male mice, MK8722 did not significantly decrease mechanical hypersensitivity after plantar incision surgery, except a small effect at day 7 after incision; however, 7-day treatment did block hyperalgesic priming (Fig. 6, A-C). In the female cohorts, MK8722 again had no effect on the initial incision-induced mechanical hypersensitivity but also blocked hyperalgesic priming after
$\mathrm{PGE}_{2}$, just as with the male cohort (Fig. 6, D-F). These experiments demonstrate that direct activators of AMPK have a strong effect on hyperalgesic priming but do not influence pain hypersensitivity caused by acute incision. A separate group of male mice were allocated into MK8722 and vehicle treatment groups and baselined for von Frey and the rotarod. These mice were treated for 7 days and given a sham surgery on day 3 . The mice were tested by the von Frey test throughout this time; after treatment, they were tested on the rotarod again to assess the effects of MK8722 on locomotive behavior. As with metformin and NCLS on sham mice, MK8722 had no effect on von Frey or locomotive behavior in male mice (Supplemental Fig. 5, A-D).

We have previously given two local injections of AMPK activators (one at the time of incision and another 24 hours later) to demonstrate that local activation of AMPK can
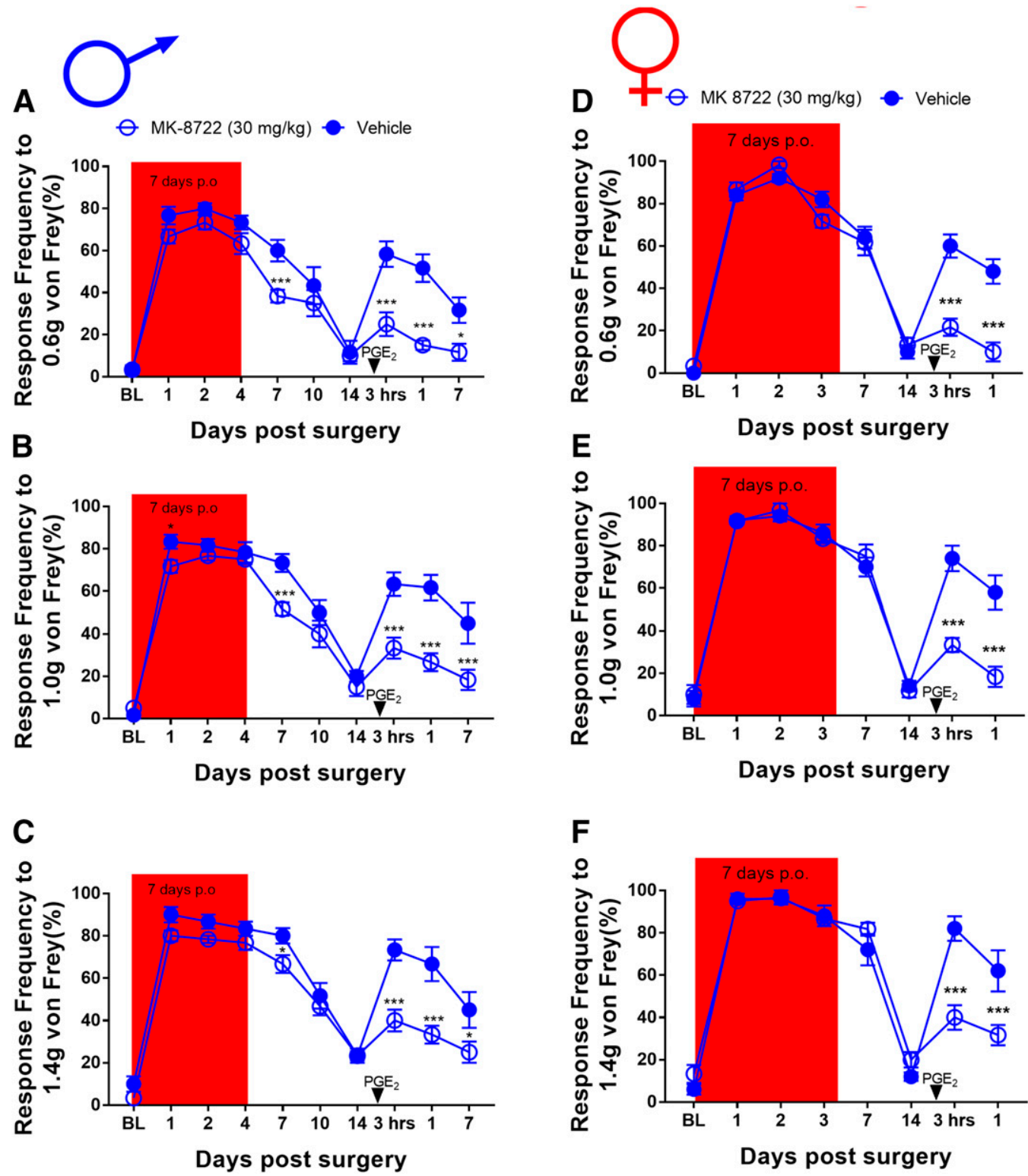

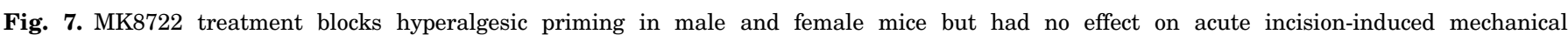

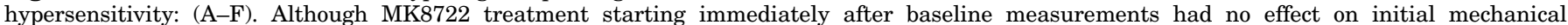

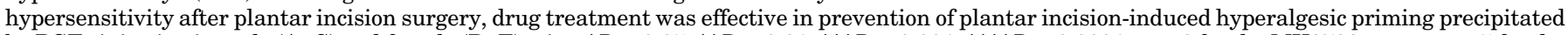

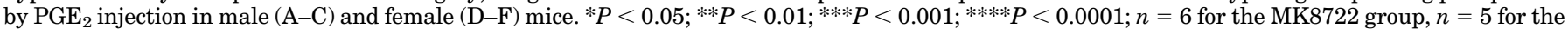
vehicle group. 
attenuate incision-evoked mechanical hypersensitivity (Tillu et al., 2012; Burton et al., 2017). We assessed whether MK8722 (10- $\mu \mathrm{g}$ dose per injection) would affect incision-evoked pain. In both male (Fig. 7, A-C) and female (Fig. 7, D-F) mice, MK8722 had no effect on acute mechanical hypersensitivity, but local treatment with this direct AMPK activator did attenuate hyperalgesic priming (Fig. 7). This effect is consistent with the systemic dosing experiment with MK8722 but is contrasts with our previous findings with local injection of indirect AMPK activators (e.g., resveratrol) that blocked acute mechanical hypersensitivity and hyperalgesic priming (Tillu et al., 2012; Burton et al., 2017).

Like NCLS, MK8722 activates AMPK in many cell types but has not previously been tested on DRG neurons. DRGs were cultured from naïve male mice and treated with vehicle, $100 \mathrm{nM}$, or $10 \mu \mathrm{M}$ MK8722 for 1 hour. We observed a significant increase in p-ACC intensity with MK8722 treatment at $100 \mathrm{nM}$ and
$10 \mu \mathrm{M}$, demonstrating that MK8722 induces AMPK activation neurons (Fig. 8, A-D). Quantification was done by measuring the maximum florescence (Fig. 8D). Based on this result, we conducted a full-concentration response curve for MK8722 and obtained an approximate $\mathrm{EC}_{50}$ of $900 \mathrm{nM}$ (95\% confidence interval, $253 \mathrm{nM}-2.62 \mu \mathrm{M}$ ) for AMPK activation in DRG neurons (Fig. 8E). This $\mathrm{EC}_{50}$ for MK8722 is similar to observations in rat primary hepatocytes for AMPK activation (Myers et al., 2017).

Plantar Incision Decreases pAMPK in the DRG Ipsilateral to Incision Injury. Male mice went through the same behavioral battery as already described, and then plantar incision surgery was performed. Two hours postsurgery, lumbar DRGs were taken on the ipsilateral and contralateral sides from these mice and homogenized for Western blotting. We used p-AMPK and total-AMPK as the primary antibodies to assess the effects of injury on AMPK signaling. We saw
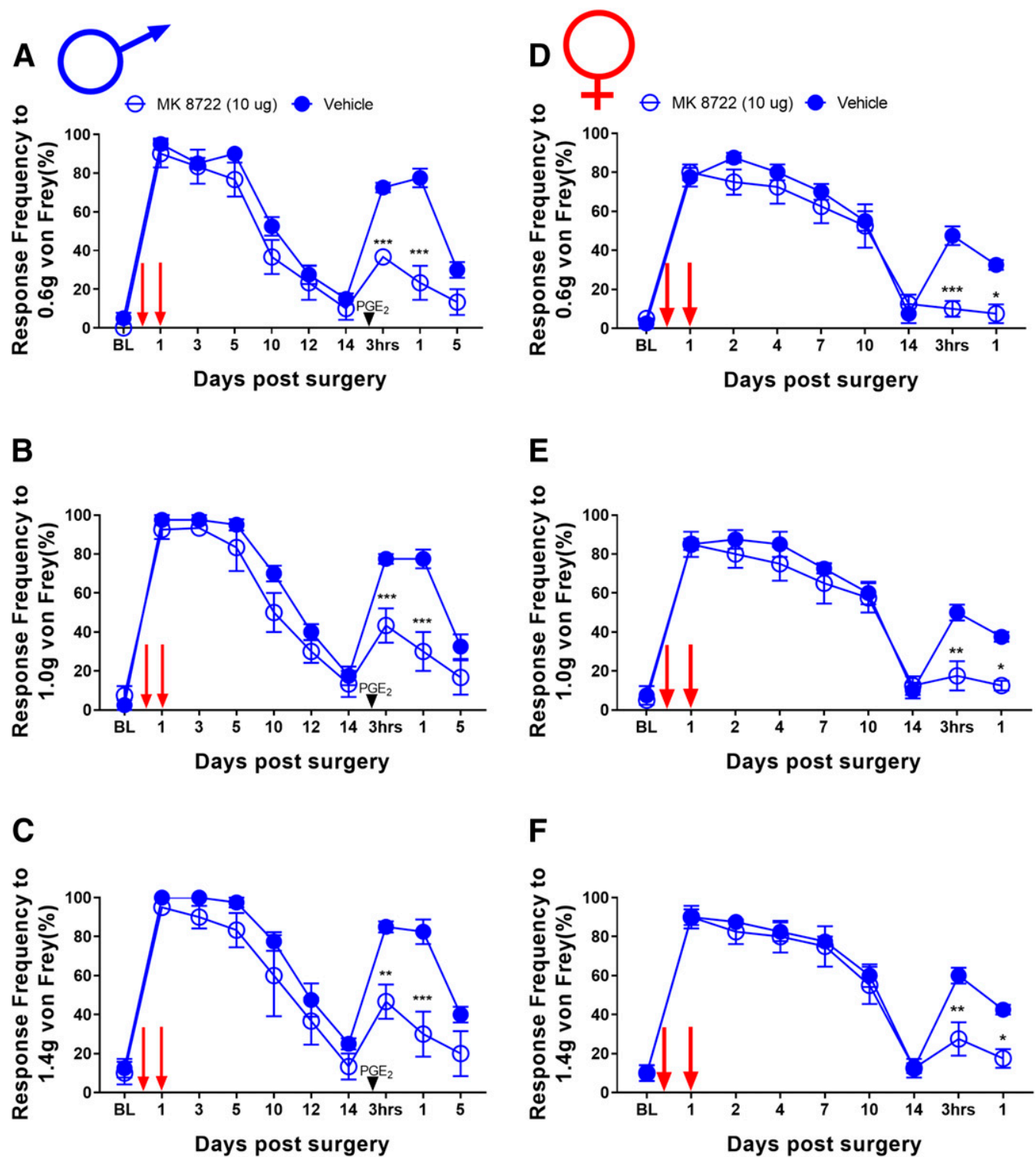

Fig. 8. Local MK8722 treatment attenuates hyperalgesic priming in male and female mice but had no effect on acute incision-induced mechanical hypersensitivity: Local injection of MK8722 at the time of incision and again 24 hours later (red arrows) had no effect on initial mechanical hypersensitivity after plantar incision surgery in male or female mice; however, drug treatment was effective in prevention of plantar incision-induced hyperalgesic priming precipitated by $\mathrm{PGE}_{2}$ injection in male (A-C) and female (D-F) mice. $* P<0.05$; $* * P<0.01 ; * * * P<0.001 ; n=4$ for the MK8722 group, $n=4$ for the vehicle group in both male and female mice. 
a significant decrease in p-AMPK/total-AMPK ratio in ipsilateral DRGs compared with contralateral DRGs (Figs. 9 and 10). This finding is consistent with previously published data demonstrating that pain-inducing stimuli cause a decrease in AMPK signaling in DRG (Atef et al., 2019). Given that many direct AMPK activators require AMPK phosphorylation for their pharmacologic activity, this potentially explains why those compounds are less efficacious for acute incisional pain.

\section{Discussion}

One of the key findings of this study is that whereas metformin's sex differential effect on neuropathic pain (Inyang et al., 2019) can also be observed in the incisional pain model, other AMPK activators did not have a sex-specific effect on pain in our experiments. NCLS, ZLN-024, and MK 8722 all had the same level of efficacy on hyperalgesic priming in male and female mice. Metformin had a robust initial antihyperalgesic effect and blocked hyperalgesic priming in male mice but showed no effect in female mice. These sex differences are not readily explained by the pharmacokinetics of metformin. In fact, female mice had higher plasma and brain levels of metformin than did male mice. Although we do not have an explanation for the sex differences in metformin's efficacy in incisional or neuropathic pain models in mice, our work does illustrate that other AMPK activators can be effective in the incisional model in both sexes. A possible mechanism that can be explored in future work is sexual
A

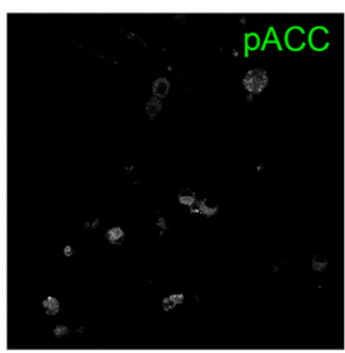

B

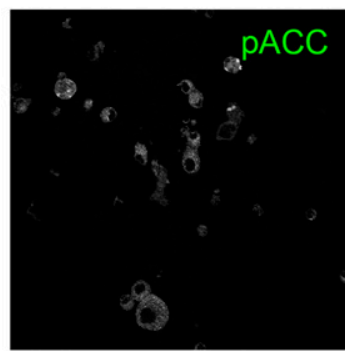

C

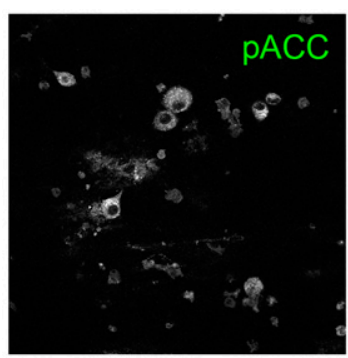

D

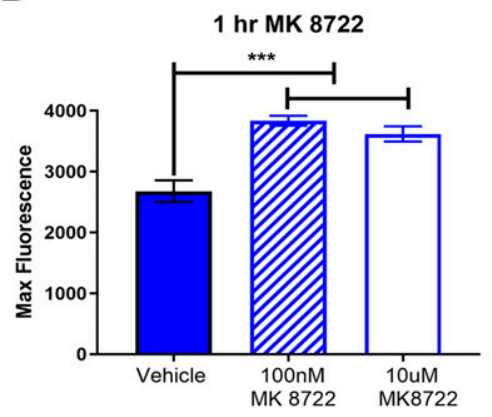

Vehicle

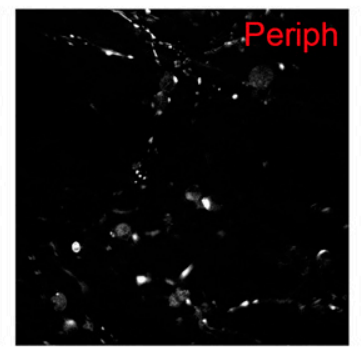

100 nM MK 8722

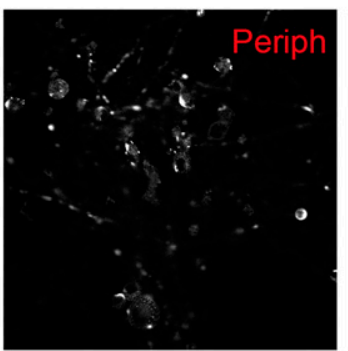

10 uM MK 8722
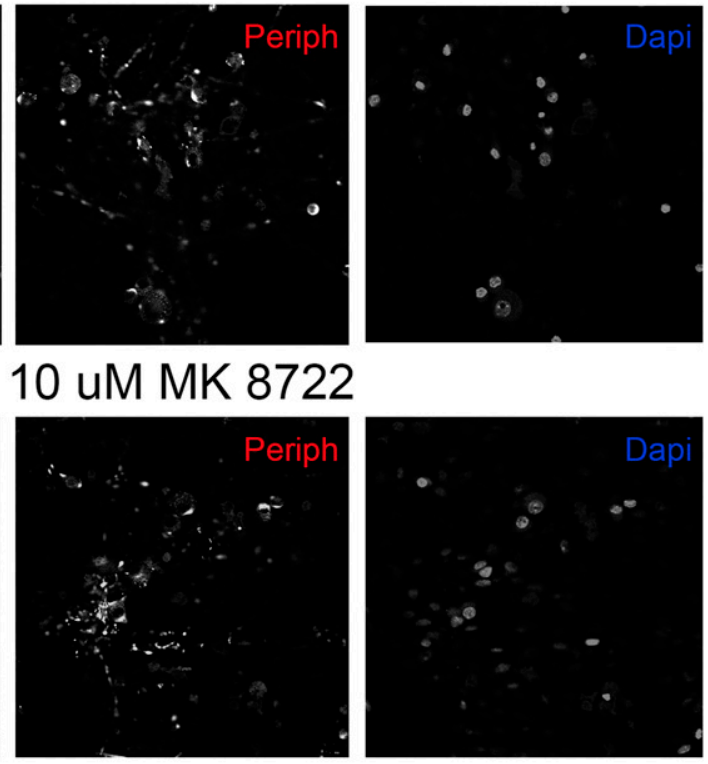

E

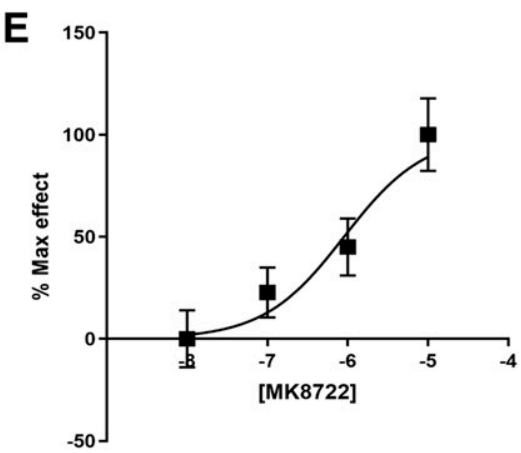

Fig. 9. MK8722 induces AMPK activation in DRG neurons in vitro. Neuron cultures were treated with vehicle (A), 100 $\mathrm{nM}(\mathrm{B})$, or $10 \mu \mathrm{M}$ MK 8722 (C) for 1 hour Representative immunohistochemistry images of the DRG neurons at $40 \times$ magnification. Quantification of images shown in (D). 100 $\mathrm{nM}$ and $10 \mu \mathrm{M}$ NCLS increased p-ACC intensity in male neuron cultures. Only neurons positive for peripherin staining were analyzed. Maximum florescence refers to the maximum florescence intensity per neuron analyzed. ** $P<0.01$; *** $P<0.001 . n=39$ images analyzed per group. (E) Shows a full concentration-response curve of MK8722 with an approximate $\mathrm{EC}_{50}$ of $900 \mathrm{nM}$ (95\% confidence interval, $253 \mathrm{nM}-2.62 \mu \mathrm{M})$. 


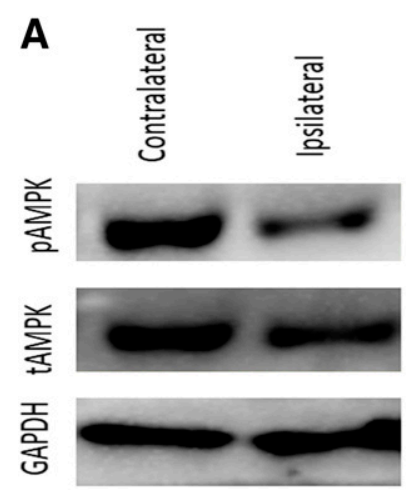

\section{B}

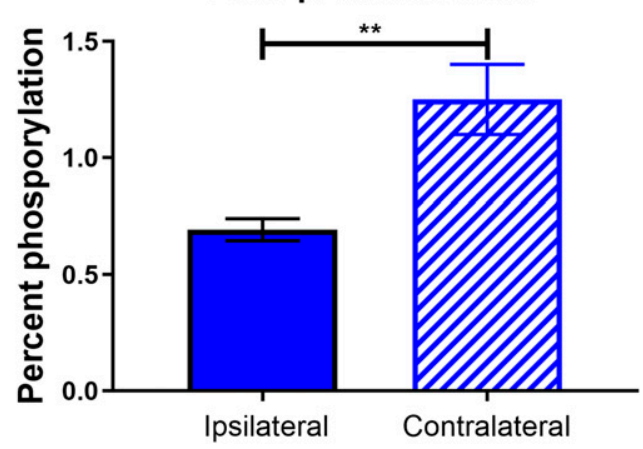

Fig. 10. Incision decreases AMPK signaling in injured lumbar DRGs: (A) Plantar incision caused a decrease in p-AMPK signaling in ipsilateral lumbar DRGs 2 hours postsurgery measured by Western blot. (B) Incision decreased the ratio between p-AMPK and total-AMPK in the ipsilateral DRGs compared with the side contralateral from the injury. ${ }^{* *} P<0.01$; students $t$ test; $N=5$ per condition in male mice. dimorphic expression of transporters that are required for metformin to enter cells. Previous studies have demonstrated that this is an important aspect of metformin's action and is controlled by sex hormones (Cai et al., 2019).

Another key finding of this study is our demonstration that indirect AMPK activators are more efficacious in the acute phase of mechanical hypersensitivity after incisional injury. Whereas the positive allosteric modulators ZLN-024 and MK 8722 showed a robust ability to block hyperalgesic priming, these drugs did little to attenuate the initial mechanical hypersensitivity caused by incision pain. In contrast, pretreatment with NCLS (in both males and females) and metformin (in males) caused a large initial antihyperalgesic effect and more rapid resolution of surgery-induced hypersensitivity in addition to blocking priming. Despite this behavioral difference, we did not see a difference in vitro in the ability of these compounds to activate AMPK. Both NCLS and MK 8722 induced significant AMPK activation in DRG neurons culture with an $\mathrm{EC}_{50}$ for $\mathrm{MK} 8722$ that was consistent with values previously reported in the literature (Myers et al., 2017). It is unlikely that these differences occurred from pharmacokinetic or pharmacodynamic issues in the DRG because we used these compounds at in vivo doses that have been thoroughly vetted previously for AMPK activation. In line with this, we show here that NCLS activates AMPK in the DRG, but not liver, of male and female mice.

How, then, can the difference in acute effects of these different modes of AMPK activation be explained? One potential explanation for the behavioral difference we saw between the indirect AMPK activators and positive allosteric modulators is the decrease in pAMPK in the DRG caused by incision injury, which has been shown previously in diabetic neuropathic pain (Atef et al., 2019). A decrease in DRG neuron levels of phosphorylated AMPK would theoretically substantially decrease the ability of allosteric modulators to activate AMPK. This decrease is because these drugs are thought to rely on phosphorylated AMPK to be able to increase AMPK activity. If AMPK phosphorylation is decreased by a preceding injury, these drugs would achieve a smaller amount of enhanced AMPK activation unless upstream AMPK phosphorylation is increased via some other mechanism. On the other hand, indirect AMPK activators increase AMPK phosphorylation because they act via upstream kinases that then phosphorylate AMPK. A limitation of this idea is that the existing data on MK8722 has not directly assessed whether AMPK phosphorylation is needed for AMPK activation with this compound, but it is clearly a direct activator of the enzyme (Myers et al., 2017).

As previously mentioned, metformin activates AMPK indirectly through the upstream kinase LKB1 (Shaw et al., 2005). NCLS is thought to increase AMPK phosphorylation via a cAMP- and ADP/ATP ratio-dependent mechanism, which may act similarly to resveratrol (Julien et al., 2017). Importantly, resveratrol also inhibits acute incision-evoked pain and blocks the development of hyperalgesic priming (Burton et al., 2017). Therefore, from the perspective of therapeutic development of AMPK activators for the treatment of postsurgical pain, our findings support a focus on the potent indirect AMPK activators. Given that NCLS is far more potent than metformin, this molecule may represent an excellent starting point for further refinement in this space.

Another question arising from our work is why direct allosteric modulators of AMPK are effective in blocking the development of hyperalgesic priming but not acute incisionevoked hypersensitivity. Previous studies have made it clear that hyperalgesic priming can be completely reversed without having any effect at all on the acute pain phase (Asiedu et al., 2011; Price and Inyang, 2015) as we have shown here with ZLN024 and MK8722. Although we have not investigated this finding directly, we favor the hypothesis that these compounds block mTOR and MAPK-dependent protein synthesis sufficiently to attenuate gene expression of proteins that are needed for the development of hyperalgesic priming. This could include the synthesis of proteins, like CREB, that act as retrograde signaling factors from the site of injury to then regulate transcriptional programs that are needed for the transition to a chronic pain state (Melemedjian et al., 2014). Our findings suggest that inhibition of translation of these proteins requires less AMPK activation than does the translation of proteins required for the acute sensitization of nociceptors. This hypothesis can be explored in future studies.

Several shortcomings of our study should be addressed in the future. First, although we have done numerous pharmacologic manipulations in this study, we have not used genetic approaches to determine directly whether AMPK is responsible for the effects we have observed. Second, NCLS's pharmacology is potentially complex and may involve signaling pathways that are independent of AMPK. We cannot rule out this possibility based on our current experiments. Another caveat is that we have relied on evoked testing for pain assessment and have not used other methods, such as paw guarding or mouse grimace scale. Our future work will focus on nonevoked pain measures. Finally, whether the 
hyperalgesic priming paradigm truly models development of chronic postsurgical pain is controversial; however, we are not aware of a widely accepted model of chronic postsurgical pain.

In conclusion, direct and indirect AMPK activators block the transition of acute pain to chronic pain that is potentially modeled preclinically by the hyperalgesic priming paradigm, and they can do this in both male and female mice; however, indirect AMPK activators have an acute antihyperalgesic effect that is not seen with direct positive allosteric modulators. Based on these findings, we conclude that indirect AMPK activators, like NCLS, should be further pursued as a treatment option for postsurgical pain that could achieve analgesia and a blockade of development of chronic pain in both sexes. More work is needed to determine the selectivity of NCLS at AMPK; but, given the favorable pharmacologic profile that has already been described for this compound, we propose that it has excellent potential as a starting point for further development.

\section{Acknowledgments}

We thank members of the Price, Dussor, and Burton laboratories for input throughout this project.

\section{Authorship Contributions}

Participated in research design: Inyang, Burton, Dussor, Price.

Conducted experiments: Inyang, Szabo-Pardi, Wentworth, McDougal, Ramirez, Pradhan.

Performed data analysis: Inyang, Burton, Price.

Wrote or contributed to the writing of the manuscript: Inyang, Dussor, Price.

\section{References}

Asiedu MN, Tillu DV, Melemedjian OK, Shy A, Sanoja R, Bodell B, Ghosh S, Porreca $\mathrm{F}$, and Price TJ (2011) Spinal protein kinase $\mathrm{M} \zeta$ underlies the maintenance mechanism of persistent nociceptive sensitization. J Neurosci 31:6646-6653.

Atef MM, El-Sayed NM, Ahmed AAM, and Mostafa YM (2019) Donepezil improves neuropathy through activation of AMPK signalling pathway in streptozotocininduced diabetic mice. Biochem Pharmacol 159:1-10.

Banik RK, Subieta AR, Wu C, and Brennan TJ (2005) Increased nerve growth factor after rat plantar incision contributes to guarding behavior and heat hyperalgesia. Pain 117:68-76.

Banik RK, Woo YC, Park SS, and Brennan TJ (2006) Strain and sex influence on pain sensitivity after plantar incision in the mouse. Anesthesiology 105:1246-1253.

Bullón P, Alcocer-Gómez E, Carrión AM, Marín-Aguilar F, Garrido-Maraver J, Román-Malo L, Ruiz-Cabello J, Culic O, Ryffel B, Apetoh L, et al. (2016) AMPK phosphorylation modulates pain by activation of NLRP3 inflammasome. Antioxid Redox Signal 24:157-170.

Burton MD, Tillu DV, Mazhar K, Mejia GL, Asiedu MN, Inyang K, Hughes T, Lian B, Dussor G, and Price TJ (2017) Pharmacological activation of AMPK inhibits incision-evoked mechanical hypersensitivity and the development of hyperalgesic priming in mice. Neuroscience 359:119-129.

Cai H, Everett RS, and Thakker DR (2019) Efficacious dose of metformin for breast cancer therapy is determined by cation transporter expression in tumours. $\mathrm{Br}$ $J$ Pharmacol 176:2724-2735

Cao C, Huang W, Zhang N, Wu F, Xu T, Pan X, Peng C, and Han B (2018) Narciclasine induces autophagy-dependent apoptosis in triple-negative breast cancer cells by regulating the AMPK-ULK1 axis (Abstract). Cell Prolif 51:e12518.

Dumont P, Ingrassia L, Rouzeau S, Ribaucour F, Thomas S, Roland I, Darro F, Lefranc F, and Kiss R (2007) The Amaryllidaceae isocarbostyril narciclasine induces apoptosis by activation of the death receptor and/or mitochondrial pathways in cancer cells but not in normal fibroblasts. Neoplasia 9:766-776.

Feng D, Biftu T, Romero FA, Kekec A, Dropinski J, Kassick A, Xu S, Kurtz MM, Gollapudi A, Shao Q, et al. (2017) Discovery of MK-8722: a systemic, direct panactivator of AMP-activated protein kinase. ACS Med Chem Lett 9:39-44.

Finnerup NB, Attal N, Haroutounian S, McNicol E, Baron R, Dworkin RH, Gilron I, Haanpää M, Hansson P, Jensen TS, et al. (2015) Pharmacotherapy for neuropathic pain in adults: a systematic review and meta-analysis. Lancet Neurol 14:162-173.

Hardie DG (2007) AMP-activated/SNF1 protein kinases: conserved guardians of cellular energy. Nat Rev Mol Cell Biol 8:774-785.
Haroutiunian S, Nikolajsen L, Finnerup NB, and Jensen TS (2013) The neuropathic component in persistent postsurgical pain: a systematic literature review. Pain 154:95-102.

Institute of Medicine Committee on Advancing Pain Research, Care, and Education (2011) The National Academies Collection: reports funded by National Institutes of Health, in Relieving Pain in America: A Blueprint for Transforming Prevention, Care, Education, and Research, National Academies Press (US) National Academy of Sciences, Washington (DC).

Inyang KE, Szabo-Pardi T, Wentworth E, McDougal TA, Dussor G, Burton MD, and Price TJ (2019) The antidiabetic drug metformin prevents and reverses neuropathic pain and spinal cord microglial activation in male but not female mice. Pharmacol Res 139:1-16.

Julien SG, Kim SY, Brunmeir R, Sinnakannu JR, Ge X, Li H, Ma W, Yaligar J, Kn BP, Velan SS, et al. (2017) Narciclasine attenuates diet-induced obesity by promoting oxidative metabolism in skeletal muscle (Abstract). PLoS Biol 15:e1002597.

Kahn BB, Alquier T, Carling D, and Hardie DG (2005) AMP-activated protein kinase: ancient energy gauge provides clues to modern understanding of metabolism. Cell Metab 1:15-25.

Kehlet H, Jensen TS, and Woolf CJ (2006) Persistent postsurgical pain: risk factors and prevention. Lancet 367:1618-1625.

Lubahn C, Schaller JA, Shewmacker E, Wood C, Bellinger DL, Byron D, Melody N Pettit GR, and Lorton D (2012) Preclinical efficacy of sodium narcistatin to reduce inflammation and joint destruction in rats with adjuvant-induced arthritis. Rheumatol Int 32:3751-3760.

Mejia GL, Asiedu MN, Hitoshi Y, Dussor G, and Price TJ (2016) The potent, indirect adenosine monophosphate- activated protein kinase activator R419 attenuates mitogen-activated protein kinase signaling, inhibits nociceptor excitability, and reduces pain hypersensitivity in mice (Abstract). Pain Rep 1:e562.

Melemedjian OK, Asiedu MN, Tillu DV, Sanoja R, Yan J, Lark A, Khoutorsky A, Johnson J, Peebles KA, Lepow T, et al. (2011) Targeting adenosine monophosphate-activated protein kinase (AMPK) in preclinical models reveals a potential mechanism for the treatment of neuropathic pain (Abstract). Mol Pain 7:70

Melemedjian OK, Tillu DV, Moy JK, Asiedu MN, Mandell EK, Ghosh S, Dussor G, and Price TJ (2014) Local translation and retrograde axonal transport of CREB regulates IL-6-induced nociceptive plasticity (Abstract). Mol Pain 10:45.

Myers RW, Guan HP, Ehrhart J, Petrov A, Prahalada S, Tozzo E, Yang X, Kurtz MM, Trujillo M, Gonzalez Trotter D, et al. (2017) Systemic pan-AMPK activator MK8722 improves glucose homeostasis but induces cardiac hypertrophy. Science $\mathbf{3 5 7}$ : 507-511.

Price TJ, Basbaum AI, Bresnahan J, Chambers JF, De Koninck Y, Edwards RR, Ji RR, Katz J, Kavelaars A, Levine JD, et al. (2018) Transition to chronic pain: opportunities for novel therapeutics. Nat Rev Neurosci 19:383-384.

Price TJ and Inyang KE (2015) Commonalities between pain and memory mechanisms and their meaning for understanding chronic pain. Prog Mol Biol Transl Sci 131:409-434.

Qin X, Qiao H, Wu S, Cheng J, Wan Q, and Liu R (2018) Curcumin inhibits monocyte chemoattractant protein-1 expression in TNF- $\alpha$ induced astrocytes through AMPK pathway. Neurochem Res 43:775-784.

Rena G, Hardie DG, and Pearson ER (2017) The mechanisms of action of metformin Diabetologia 60:1577-1585.

Sato Y and Ohshima T (2000) The expression of mRNA of proinflammatory cytokines during skin wound healing in mice: a preliminary study for forensic wound age estimation (II). Int J Legal Med 113:140-145.

Shaw RJ, Lamia KA, Vasquez D, Koo SH, Bardeesy N, Depinho RA, Montminy M, and Cantley LC (2005) The kinase LKB1 mediates glucose homeostasis in liver and therapeutic effects of metformin. Science 310:1642-1646.

Song H, Han Y, Pan C, Deng X, Dai W, Hu L, Jiang C, Yang Y, Cheng Z, Li F, et al. (2015) Activation of adenosine monophosphate-activated protein kinase suppresses neuroinflammation and ameliorates bone cancer pain: involvement of inhibition on mitogen-activated protein kinase. Anesthesiology 123:1170-1185.

Tillu DV, Melemedjian OK, Asiedu MN, Qu N, De Felice M, Dussor G, and Price TJ (2012) Resveratrol engages AMPK to attenuate ERK and mTOR signaling in sensory neurons and inhibits incision-induced acute and chronic pain (Abstract). Mol Pain 8:5.

Weihrauch M and Handschin C (2018) Pharmacological targeting of exercise adaptations in skeletal muscle: benefits and pitfalls. Biochem Pharmacol 147:211-220. Wu W, Wang S, Liu Q, Shan T, and Wang Y (2018) Metformin protects against LPSinduced intestinal barrier dysfunction by activating AMPK pathway. Mol Pharm 15:3272-3284.

Yui S, Mikami M, Mimaki Y, Sashida Y, and Yamazaki M (2001) [Inhibition effect of Amaryllidaceae alkaloids, lycorine and lycoricidinol on macrophage TNF-alpha production]. Yakugaku Zasshi 121:167-171.

Zhang BB, Zhou G, and Li C (2009) AMPK: an emerging drug target for diabetes and the metabolic syndrome. Cell Metab 9:407-416.

Zhang LN, Xu L, Zhou HY, Wu LY, Li YY, Pang T, Xia CM, Qiu BY, Gu M, Dong TC, et al. (2013) Novel small-molecule AMP-activated protein kinase allosteric activator with beneficial effects in $\mathrm{db} / \mathrm{db}$ mice (Abstract). PLoS One 8:e72092.

Address correspondence to: Theodore J. Price, School of Behavioral and Brain Sciences, University of Texas at Dallas, BSB 14.102, $800 \mathrm{~W}$ Campbell Rd, Richardson TX 75080. E-mail: Theodore.price@utdallas.edu 